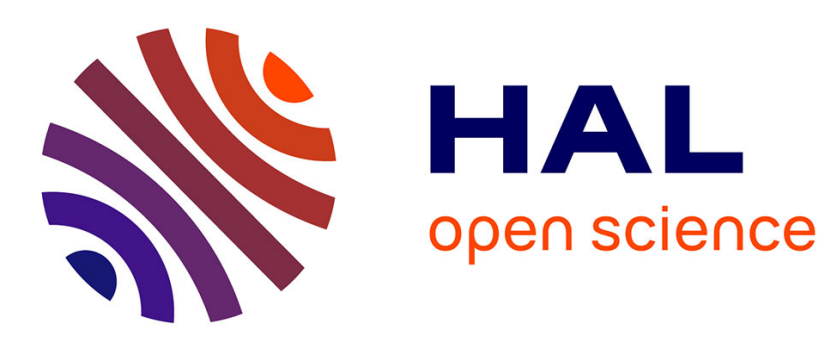

\title{
Reconstruction of HMBC Correlation Networks: A Novel NMR-based Contribution to Metabolite Mixture Analysis
}

Ali Bakiri, Jane Hubert, Romain Reynaud, Carole Lambert, Agathe Martinez, Jean- Hugues Renault, Jean-Marc Nuzillard

\section{To cite this version:}

Ali Bakiri, Jane Hubert, Romain Reynaud, Carole Lambert, Agathe Martinez, et al.. Reconstruction of HMBC Correlation Networks: A Novel NMR-based Contribution to Metabolite Mixture Analysis. Journal of Chemical Information and Modeling, 2018, 58 (2), pp.262-270. 10.1021/acs.jcim.7b00653 . hal-01692926

\section{HAL Id: hal-01692926 \\ https://hal.univ-reims.fr/hal-01692926}

Submitted on 13 Sep 2021

HAL is a multi-disciplinary open access archive for the deposit and dissemination of scientific research documents, whether they are published or not. The documents may come from teaching and research institutions in France or abroad, or from public or private research centers.
L'archive ouverte pluridisciplinaire HAL, est destinée au dépôt et à la diffusion de documents scientifiques de niveau recherche, publiés ou non, émanant des établissements d'enseignement et de recherche français ou étrangers, des laboratoires publics ou privés. 
4 Ali Bakiri, ${ }^{1,2}$ Jane Hubert, ${ }^{1,}{ }^{*}$ Romain Reynaud, ${ }^{2}$ Carole Lambert, ${ }^{2}$ Agathe Martinez, ${ }^{1}$ Jean-

$5 \quad$ Hugues Renault, ${ }^{1}$ and Jean-Marc Nuzillard ${ }^{1}$

6

$7 \quad{ }^{1}$ Institut de Chimie Moléculaire de Reims, UMR CNRS 7312, SFR CAP'SANTE, Université de

8 Reims Champagne-Ardenne, Reims, France

$9 \quad{ }^{2}$ Givaudan France, Active Beauty Department, Pomacle, France

10

11

*e-mail: jane.hubert@univ-reims.fr 


\section{Abstract}

2 Here is introduced a new in silico method for the dereplication of natural metabolite mixtures

3 based on $\mathrm{HMBC}$ and $\mathrm{HSQC}$ spectra that inform about short-range and long-range $\mathrm{H}-\mathrm{C}$

4 correlations occurring in the carbon skeleton of individual chemical entities. Starting from the

$5 \mathrm{HMBC}$ spectrum of a metabolite mixture, an algorithm was developed in order to recover

6 individualized $\mathrm{HMBC}$ footprints of the mixture constituents. The collected $\mathrm{H}-\mathrm{C}$ correlations are

7 represented by a network of NMR peaks connected each other when sharing either a ${ }^{1} \mathrm{H}$ or

$8{ }^{13} \mathrm{C}$ chemical shift value. The obtained network is then divided into clusters using a community

9 detection algorithm, and finally each cluster is tentatively assigned to a molecular structure by mean of a NMR chemical shift database containing the theoretical HMBC and HSQC

11 correlation data of a range of natural metabolites. The proof of principle of this method is demonstrated on a model mixture of 3 known natural compounds and then on a real-life bark extract obtained from the common spruce (Picea abies L.). 


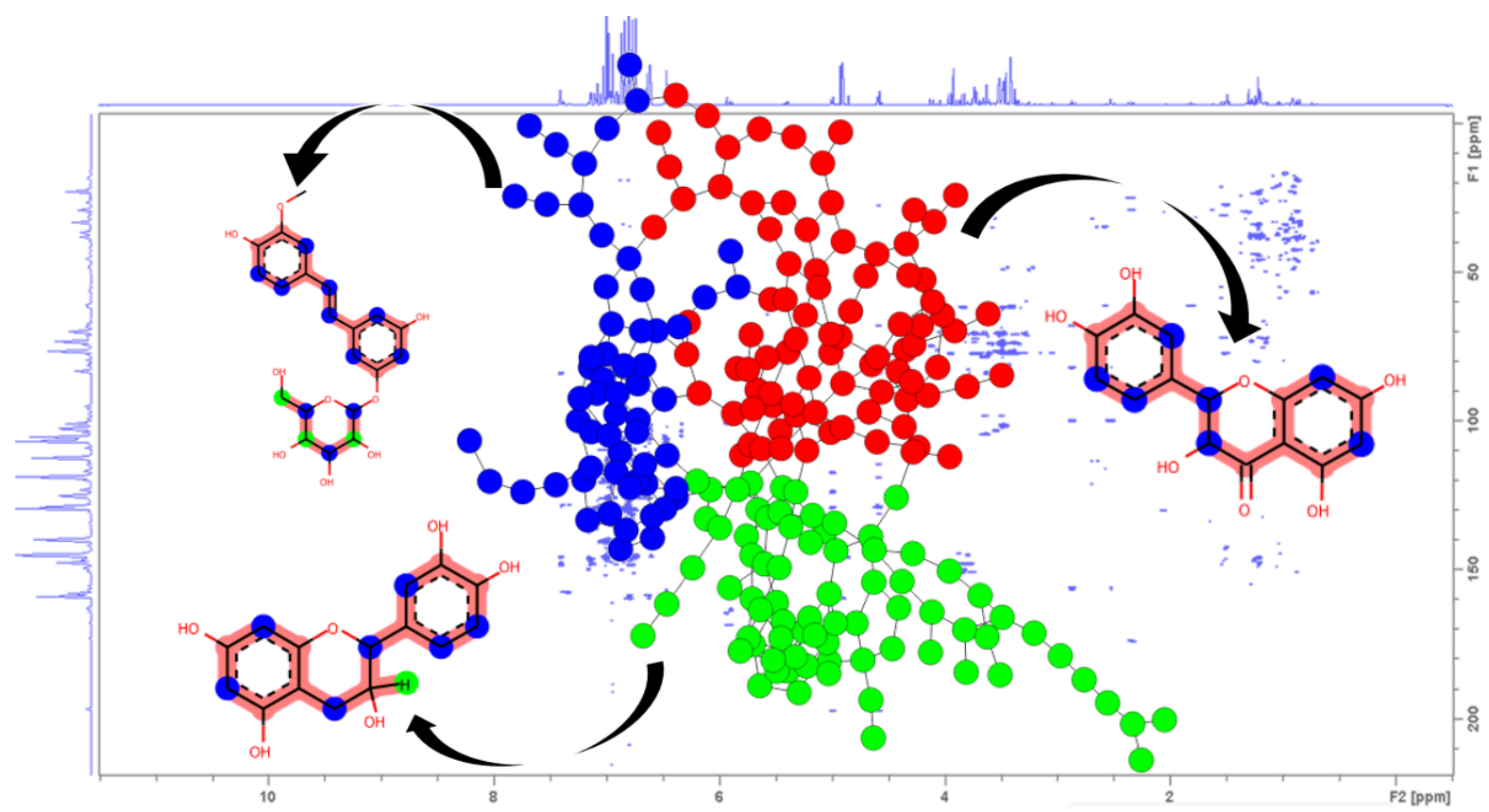

\section{INTRODUCTION}

Identification and quantification of chemical entities directly within complex mixtures is gaining a great attention in the field of natural product research and more generally in all metabolomics studies dealing with the analysis of low molecular weight metabolites in biological systems (Wist, 2016)(Gaudêncio \& Pereira, 2015)(Bingol et al., 2016) (Kim, Choi, \& Verpoorte, 2011). Over the last decade, a range of new analytical strategies have emerged to accelerate metabolite identification processes, mainly based on the so-called dereplication concept (Beutler, Alvarado, Schaufelberger, Andrews, \& McCloud, 1990)ref revue wolfender (Gaudêncio \& Pereira, 2015; Hubert, Nuzillard, \& Renault, 2015). Among the panel of analytical techniques available, Nuclear Magnetic Resonance (NMR) remains by far the most efficient method to unambiguously identify small organic molecules (Smolinska, Blanchet, Buydens, \& Wijmenga, 2012)(Schripsema, 2010). NMR has the unique ability to probe 
1 structural information at the atom level for NMR-active nuclei such as ${ }^{1} \mathrm{H},{ }^{13} \mathrm{C},{ }^{15} \mathrm{~N}$ and ${ }^{31} \mathrm{P}$.

2 Proton $\left({ }^{1} \mathrm{H}\right.$ nucleus) NMR spectroscopy is mostly used due to its high sensitivity, but suffers

3 from frequent signal overlaps which confuse data interpretation when dealing with samples

4 of complex composition. Conversely, ${ }^{13} \mathrm{C}$ NMR is less sensitive than ${ }^{1} \mathrm{H}$ NMR, nonetheless it

5 provides a larger chemical shift dispersion $(240 \mathrm{ppm})$ that reduces signal overlaps and leads

6 to well-resolved spectra containing individualized narrow peaks. Along with the ability to

7 obtain information about the carbon skeleton of all detected organic molecules, these

8 advantages have progressively prompted ${ }^{13} \mathrm{C}$ NMR in the core of recent dereplication

9 procedures ( Clendinen et al, 2014; Laude et al, 1986; Bighelli et al, 1994; Hubert et al, 2014).

In addition to ${ }^{1} \mathrm{H}$ and ${ }^{13} \mathrm{C}$ NMR, 2D NMR spectra such as $\mathrm{HMBC}$ (Heteronuclear Multiple Bond

Correlation spectroscopy) and HSQC (Heteronuclear Single Quantum Correlation

spectroscopy) are extremely informative. Both provide important information about atom connectivity. Such spectra have also attracted an increasing interest in recent dereplication and metabolomics studies (Bingol \& Bruschweiler, 2013 + ref pauli hsqc mask actae triterpenes)

The second breakthrough in mixture analysis strategies is the application of mathematical treatments to facilitate the interpretation of complex analytical data (Wiklund, n.d.). Along with the multivariate statistical analyses classically used in metabolomics, new mathematical methods such as data fusion (Acar, Bro, \& Smilde, 2015) and graph theory are more and more applied to rise up the relevance of complex mixture studies (Garg et al., 2015). For instance, molecular networking has recently emerged in the context of mass spectrometry (MS) as an efficient way to handle the complexity of MS/MS data. It relies on the observation that structurally similar metabolites share similar MS/MS fragmentation patterns. On the basis of this property, the correlations between MS/MS spectral data of a natural sample are 
measured and used to construct a molecular network in which each cluster of similar fragments represents a specific group of structurally close metabolites. (Winnikoff, Glukhov, Watrous, Dorrestein, \& Gerwick, 2014) (Allard et al., 2016; Yang et al., n.d.)

Here is presented a new HMBC-based dereplication method that uses a networking approach for the deconvolution of complex NMR spectra of metabolite mixtures. This method exploits the ability of $\mathrm{HMBC}$ experiments to provide connectivity information between ${ }^{1} \mathrm{H}$ and ${ }^{13} \mathrm{C}$ atoms located at their vicinity. Starting from the HMBC spectrum of a metabolite mixture, the strategy aims to build the network of ${ }^{1} \mathrm{H}-{ }^{13} \mathrm{C} \mathrm{HMBC}$ correlations and to highlight individualized patterns representing specific molecular fragments or even whole chemical structures. The isolated patterns are then identified by database search.

The proof of concept of this HMBC-based atom networking approach is demonstrated here on a synthetic model mixture of 3 known natural compounds: (+)-catechin, taxifolin and $E$ isorhapontin, and then on a genuine extract obtained from the bark extract of Picea abies.

\section{METHODS}

Sample preparation. The first analyzed sample was a model mixture composed of $3.9 \mathrm{mg}$ of taxifolin, $0.4 \mathrm{mg}$ of $(+)$-catechin, and $0.7 \mathrm{mg}$ of E-isorhapontin (all purchased from SigmaAldrich, Steinheim, Germany). The three compounds were directly dissolved in $550 \mu \mathrm{L}$ of deuterated dimethyl sulfoxide (DMSO-d6, Eurisotop, Saint-Aubin, France). The second sample was a crude methanol extract obtained from the barks of $P$. abies, prepared as described previously (Angelis et al., 2016). Briefly, 2 kg of barks were collected in the French ChampagneArdenne territory in 2014 within the context of forestry activities (Voucher specimen JH-20149, Faculty of Pharmacy, University of Reims Champagne-Ardenne, France). After drying at 
$130^{\circ} \mathrm{C}$ for $72 \mathrm{~h}$, the barks were powdered in a hammer mill and extracted with methanol under

2 magnetic stirring at ambient temperature for $20 \mathrm{~h}$. After filtration, methanol was evaporated

3 to dryness and an aliquot of the resulting extract $(20 \mathrm{mg})$ was dissolved in $550 \mu \mathrm{L}$ of DMSO-d6

4 for NMR analyses.

$5 \quad$ NMR analysis and peak picking. NMR analyses were performed at $298 \mathrm{~K}$ on an Avance III-600 spectrometer (Bruker, Karlsruhe, Germany) equipped with a cryoprobe optimized for ${ }^{1} \mathrm{H}$ detection and with cooled ${ }^{1} \mathrm{H},{ }^{13} \mathrm{C}$ and ${ }^{2} \mathrm{H}$ coils preamplifiers. $\mathrm{HSQC}$ and $\mathrm{HMBC}$ spectra were recorded using standard Bruker pulse programs. For the acquisition of HSQC spectra (pulse sequence hsqcedetgpsisp2.2), the size of FID was set at 1024 in the F2 dimension and 512 in the F1 dimension, and the number of scans was set at 4. For the acquisition of HMBC spectra (pulse sequence hmbcetgpl3nd), the size of FID was set at 2048 in the F2 dimension and 512 in the F1 dimension, and the number of scans was 4 . The spectra were manually phased and baseline corrected using the TOPSPIN 3.2 software (Bruker). Once the HMBC spectra recorded and processed, a semi-automated peak picking was performed to obtain the list of correlations. The peak picking was performed on the different regions of the spectrum first automatically, then manually in order to remove possibly meaningless peaks caused by thermal noise, $t_{1}$ noise, or residual ${ }^{1} \mathrm{~J}$ peak pairs. The automatic peak picking process was based on the minimum intensity set to the lowest contour level (Bruker processing window in Topspin 3.2).

Prediction of HMBC and HSQC data. A database of theoretical HMBC connectivity data of a range of natural metabolites was created as follows: the ${ }^{1} \mathrm{H}$ and ${ }^{13} \mathrm{C}$ NMR chemical shift values of $\approx 2700$ natural metabolites (October 2017 ) were calculated using the NMR Workbook Suite $\mathrm{ACD} /$ Labs software (Ontario, Canada). From these data, $\mathrm{HMBC}$ correlations (pairs of ${ }^{1} \mathrm{H}$ and 
$1 \quad{ }^{13} \mathrm{C}$ NMR chemical shifts) were generated using a locally written Python program. For each

2 molecule of the database, this program creates a list of all $\mathrm{H}-\mathrm{C}$ atom pairs that might be visible

3 in an $\mathrm{HMBC}$ spectrum, i.e. all $\mathrm{H}-\mathrm{C}$ pairs in which atoms are placed 2 or 3 bonds away. The

4 correlation between chemical shifts of atoms separated by 4 bonds are not observed in all

5 molecular structures and were therefore not taken into account. Once this list is established,

6 the corresponding predicted chemical shifts values of each atom pair are included in the

7 database (Fig. 1). In the same way, the HSQC correlations of all metabolites of the database

8 were determined for the ${ }^{1} \mathrm{H}-{ }^{13} \mathrm{C}$ atom pairs located at a distance of 1 bond. In addition to the

$9 \quad{ }^{1} \mathrm{H}$ and ${ }^{13} \mathrm{C}$ chemical shifts values, the parity of the number of $\mathrm{H}$ atoms attached to each $\mathrm{C}$ atom

10 was also considered. Thus, an additional column was added to the HSQC correlation table in

11 order to store the parity data. 
Figure 1| Construction of the theoretical 2D NMR database from the predicted ${ }^{1} \mathrm{H}$ and ${ }^{13} \mathrm{C}$

NMR chemical shifts of natural metabolites. 1. Listing of all $\mathrm{H}-\mathrm{C}$ atom pairs separated by 2 or 3 bonds. 2. Determination of the theoretical HMBC correlations list. 3. Addition of the created list to the database.

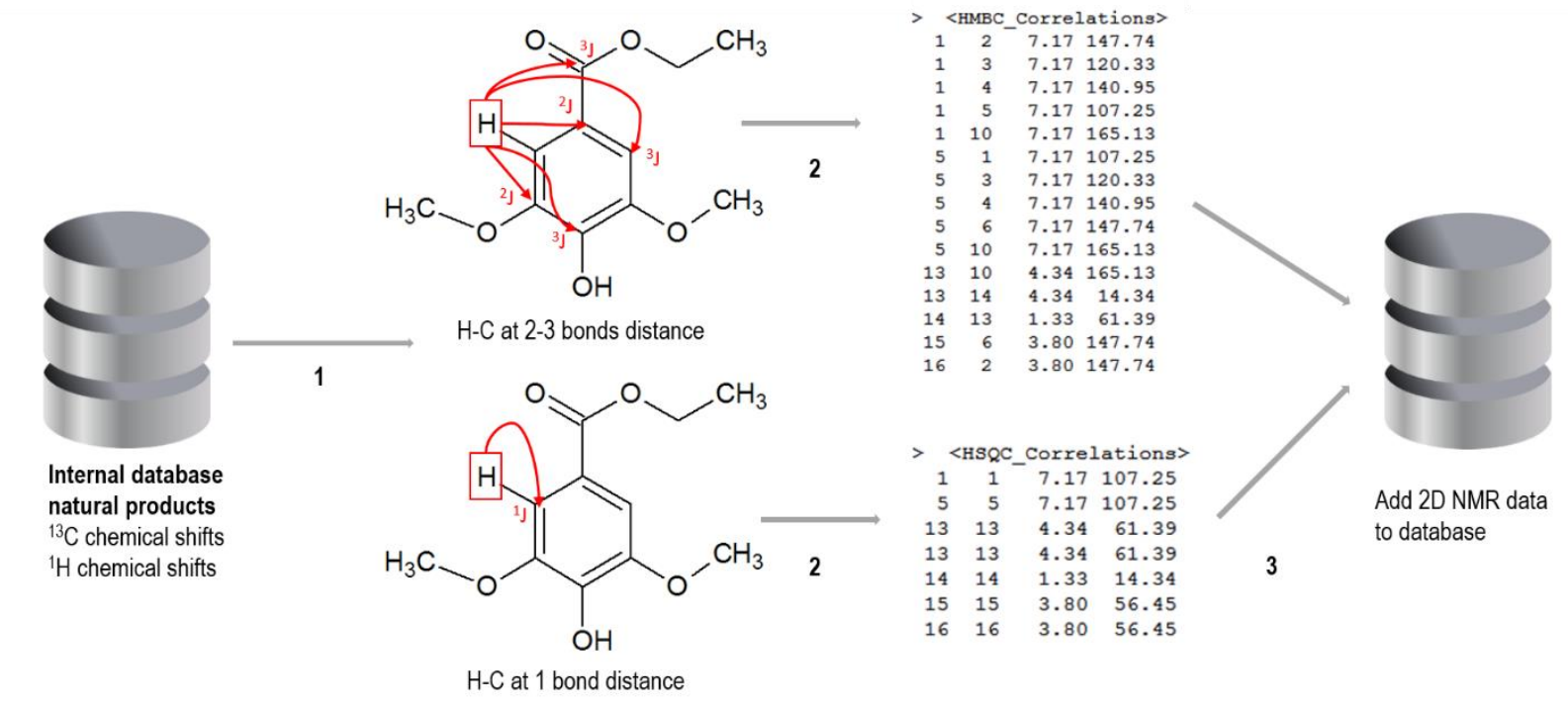

1 From experimental $\mathrm{HMBC}$ to ${ }^{1} \mathrm{H}-{ }^{13} \mathrm{C}$ chemical shift network. The ${ }^{1} \mathrm{H}-{ }^{13} \mathrm{C}$ chemical shift

2 networks were generated from the peak list of the experimental HMBC spectra using an

3 algorithm written in Python. The principle of this algorithm is to extract the HMBC correlation

4 data from the experimental HMBC peak list and then to create a network edge list by

5 connecting each peak (vertex) to its adjacent neighbors on the same row of the HMBC

6 spectrum in the $\mathrm{F} 1$ dimension (having the same ${ }^{13} \mathrm{C}$ NMR chemical shift) or on the same

7 column in the F2 dimension (having the same ${ }^{1} \mathrm{H}$ NMR chemical shift). The obtained edge list

8 is then converted to a network graph using the igraph 0.7.1 package for Python 2.7. The

9 clustering of network vertices was performed using the Louvain community detection

10 algorithm (Blondel et al.). The Louvain method is a modularity optimization-based algorithm.

11 Modularity is a quality function that measures the strength of connectivity between the nodes 
1 of the same community. It measures the density of edges inside the community compared to

2 edges outside the community. The modularity is defined as followed (J. Newman):

$$
Q=\sum\left(e_{i i}-a_{i}^{2}\right)
$$

$e_{i i}$ : The fraction of edges that connects vertices from the same group.

$e_{i j}$ : The fraction of edges that connects vertices from group $i$ to vertices from group $j$ and $a_{i}=$ $\sum \mathrm{e}_{\mathrm{ij}}$

The Louvain algorithm is a bottom-up algorithm that starts with isolated vertices as communities that will be merged progressively into meta-communities according to the gain of modularity until an optimal graph partition is reached (Blondel et al.). The "RBER" method is a variant of the Louvain algorithm that uses Erdös-Rényi null-model (Newman, M et al 2004), in which each edge has the same probability of appearing. It includes a resolution parameter that allows the control of the community's size, in general, a higher resolution parameter leads to smaller communities and vice versa. The RBER method was applied for the detection of the HMBC peak clusters in $\mathrm{HMBC}$ networks, with a resolution parameter set at 0.2 . This value was selected by trial and error and found to give the best results.

Metabolite identification. Metabolite identification was performed by comparing the theoretical $\mathrm{HMBC}$ correlations of the database metabolites to the experimental HMBC correlations of the clusters extracted by network analysis. For this purpose, an algorithm was written in Python language to obtain the lists of experimental correlations corresponding to each cluster of the HMBC correlation network. Then, these lists of correlations are compared to the theoretical HMBC correlations of database metabolites. For each theoretical HMBC correlation of the database, the algorithm attempts to find an experimental correlation that 
1 matches both ${ }^{1} \mathrm{H}$ and ${ }^{13} \mathrm{C}$ chemical shift values within a defined tolerance radius, typically set

2 at $1.5 \mathrm{ppm}$ for ${ }^{13} \mathrm{C}$ NMR chemical shifts and $0.15 \mathrm{ppm}{ }^{1} \mathrm{H}$ NMR chemical shifts. The percentage

3 of theoretical correlations that match experimental correlations for each metabolite of the database is then calculated to evaluate its likeliness to be present within the analyzed mixture. Finally, the algorithm returns a list of metabolites for each cluster, ranked in the decreasing order of matching percentage (Fig. 2). As signals belonging to a putatively identified metabolite may be divided between several clusters, a second step is then performed to compare the theoretical $\mathrm{HMBC}$ correlations of the top ranked 20 molecules of each cluster to the complete experimental list of HBMC correlations. By this way, an improved list of molecules ranked according to their decreasing percentage of matching to the whole HMBC spectrum is obtained. The resulting list is submitted to an additional validation step which consists in the comparison of the theoretical HSQC correlations of the top ranked molecules to the HSQC correlations detected experimentally. This step is performed using another locally developed algorithm which compares the ${ }^{1} \mathrm{H}$ and ${ }^{13} \mathrm{C}$ chemical shifts values of the HSQC correlations together with their parity, therefore theoretical correlations are matched to the experimental one only if both their chemical shifts and parity match. From this step, an additional score (percentage of theoretical HSQC correlations that match experimental HSQC accuracy. 
Figure 2 | HMBC atom networking workflow for metabolite mixture identification.

1

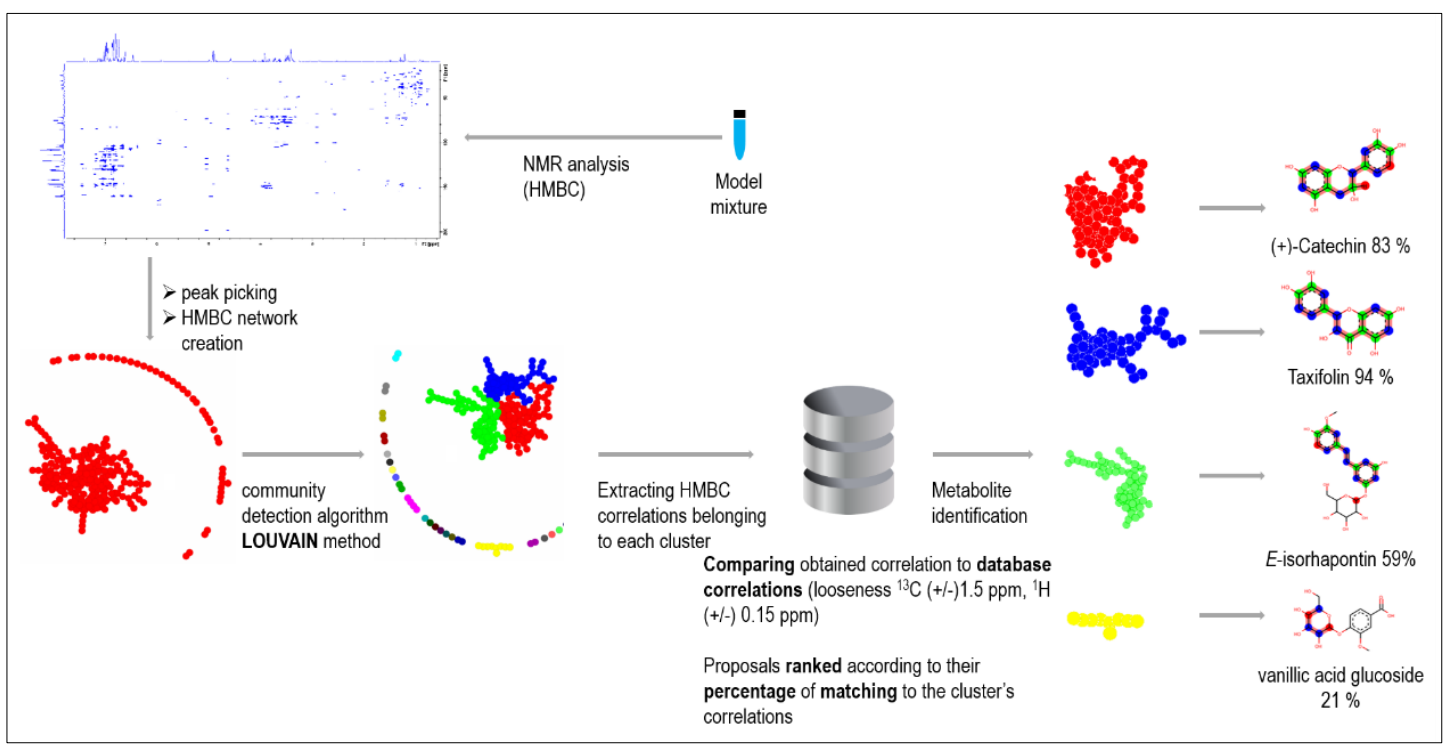

2

3

4

5

\section{RESULTS}

Proof of concept on a model mixture. A mixture of 3 commercially available compounds (2 flavonoids and 1 stilbene), taxifolin, (+)-catechin and E-isorhapontin, was used as a model in order to establish a first proof of concept of the method. The proportions of the mixture were chosen so that a molar ratio of about 10 was set between the most and the least abundant mixture components, thus giving an indication of the concentration dynamics that can possibly be handled. After NMR analysis of the mixture, a semi-automated peak picking was performed to collect all ${ }^{1} \mathrm{H}-{ }^{13} \mathrm{C}$ HMBC connectivity data from the experimental spectrum. The resulting list of ${ }^{1} \mathrm{H}$ and ${ }^{13} \mathrm{C}$ chemical shift pairs was exported as a text file and used as input to create the HMBC-derived correlation network. A network of 267 nodes representing ${ }^{1} \mathrm{H}-{ }^{13} \mathrm{C} \mathrm{HMBC}$ correlations was obtained (Fig. 3). The nodes sharing either a ${ }^{1} \mathrm{H}$ or a

${ }^{13} \mathrm{C}$ chemical shift value were connected by an edge. As the analyzed model mixture was composed of 3 molecules, the HMBC correlation network was expected to form 3 non-connected sub-networks representing each of the three molecules. However, only a big sub-network of 222 nodes was obtained, along with a small sub-network of 9 nodes and further sub-networks of 1 or 2 nodes. Non-expected 
interconnections were in fact due to the presence of wide, overlapping ${ }^{1} \mathrm{H}$ multiplet signals and of some identical chemical shift values between the three molecules. The major sub-network of 222 nodes was supposed to correspond to the three molecules of the model mixture. In an attempt to more efficiently isolate chemical shift clusters corresponding to individual molecule, a community detection algorithm was used. After the testing of several community detection approaches (Porter, Mason A et al), the Louvain method using the RBER method, which includes a resolution parameter, was found to be the most appropriate: i) the network is unweighted and undirected and the modularity based algorithms are the most appropriate to detect communities in this type of networks and ii) the resolution parameter allows the control of the communities size. By contrast, the default modularity algorithm tends to form very small communities that do not contain enough information for the identification of the metabolites. Applying this method with a resolution parameter set at 0.2 , four main clusters (C1-C4) were obtained from the initial HMBC network (Fig. 3). The experimental ${ }^{1} \mathrm{H}-$ ${ }^{13} \mathrm{C} \mathrm{HMBC}$ correlations of each cluster were then compared to the theoretical ${ }^{1} \mathrm{H}-{ }^{13} \mathrm{C} \mathrm{HMBC}$ correlations of the database metabolites. As a result, four lists of molecules having the highest likelihood to correspond to each cluster were obtained (Table 1). The proposed molecules were sorted according to their decreasing score value which represent the percentage of matching between experimental and theoretical HMBC correlations of each molecule. For cluster $\mathrm{C} 1$, the list of proposed molecules only contained flavan-3-ols (Table 1). The highest score was obtained for (+)-catechin (83\%) which was indeed one of the three constituents of the model mixture. For cluster $\mathrm{C} 2$, a list of dihydroflavonols was also obtained (Table 1), the highest score being attributed to taxifolin (94\%). For cluster C3, a list of 3 stilbenes and one tri-galloyl glucose was obtained (Table 1) with the highest score attributed to $E$ isorhapontin (54\%). This score was low as compared to the metabolites proposed for the two previous clusters, because the chemical structure of $E$-isohrapontin contains a glucose moiety and a methoxy group that do not share so many correlation with the aglycone moiety, so that the community detection algorithm affects the signals of the two moiety in separate communities. In addition, some correlations that would have allowed the connection of the communities were absent from the peak 
1 list. Cluster C4 was a small cluster containing just a few correlation values characteristic of a sugar

2 moiety (Table 1). This cluster corresponded to the glucose part of $E$-isorhapontin. A second comparison

3 was performed for the 20 top ranked molecules in each of the clusters $\mathrm{C} 1-\mathrm{C} 4$. In this case, all theoretical

$4 \quad \mathrm{HMBC}$ correlation values of the proposed molecules were compared to the whole experimental HMBC

5 peak list. This step allowed to confirm the presence of taxifolin, $(+)$-catechin and E-isorhapontin in the

6 model mixture with score values increased up to $97 \%$ for taxifolin, $93 \%$ for (+)-catechin and $70 \%$ for

7 the $E$-isorhapontin.

8 A final search was then performed to check the matching between the theoretical HSQC correlations of the top ranked molecules to the HSQC chemical shift values collected in the experimental spectrum. HSQC data inform about one-bond $\mathrm{H}-\mathrm{C}$ correlations and therefore provide information highly complementary to $\mathrm{HMBC}$ data to improve the identification accuracy. Taxifolin, (+)-catechin and E-isorhapontin achieved all an HSQC score value of $100 \%$, thus providing an additional proof of their presence in the model mixture. 
Figure 3 | HMBC-network obtained after NMR analysis of the model mixture and clustering of the vertices. Colors represent the different clusters (Red C1, Blue C2, Green C3, Yellow C4).

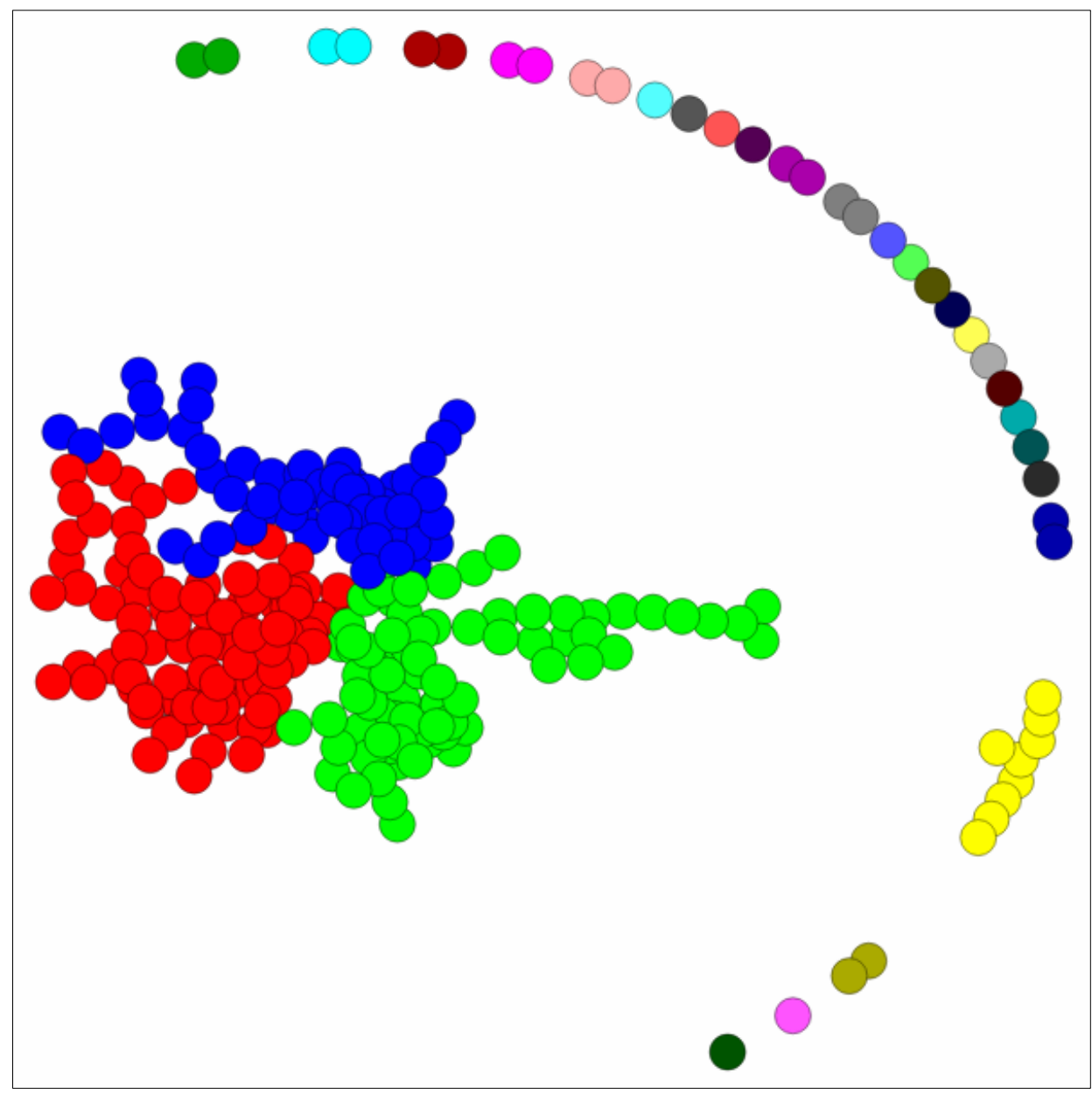


Table 1 | Proposed list of metabolites obtained for the model mixture. Colors highlight atoms for which the chemical shifts values of the experimental $\mathrm{HMBC}$ peak list matched the HMBC predicted data; Pink: matching molecular features, Blue: matching both ${ }^{1} \mathrm{H}$ and ${ }^{13} \mathrm{C}$ chemical shifts, Green: matching only ${ }^{13} \mathrm{C}$ chemical shifts, Red: Matching only ${ }^{1} \mathrm{H}$ chemical shifts.

(C1)

1 
1 Applicability to an authentic natural extract. The pertinence of the dereplication workflow based

on $\mathrm{HMBC}$ correlation networking was then evaluated on a crude methanol bark extract obtained from P. abies, a common tree growing in temperate climate regions. Fractionation by centrifugal partition chromatography as well as NMR-based chemical profiling of this extract were previously described, revealing the presence of compounds belonging to various chemical classes including stilbenes, flavonoids or phenolic acids (Angelis et al., 2016). In view of this chemical diversity, this extract was considered to be a suitable model for the testing of the real life applicability of the method. A semi-automated peak picking was performed to collect all ${ }^{1} \mathrm{H}-{ }^{13} \mathrm{C}$ HMBC connectivity data from the experimental $\mathrm{HMBC}$ spectrum of the extract. The resulting peak list was used to create a HMBC correlation network in the same way as described for the model mixture. A network of 642 nodes was obtained, containing one large sub-network of 556 nodes and two small sub-networks of 11 and 8 nodes, respectively. The other sub-networks contained less than 5 nodes. Applying the Louvain community detection algorithm to this network with the resolution parameter set at 0.2 , six clusters were obtained (C1-C6) (Fig. 4). The experimental HMBC data of these clusters were automatically compared to the theoretical $\mathrm{HMBC}$ data of the database metabolites. For Cluster C1, a list of highly similar stilbenes was obtained, the top ranked molecules of the list were $E$-resveratrol, E-piceid, $E$-isorhapontin, and $E$-astringinin with high score values of $76 \%$, $76 \%, 73 \%$ and $71 \%$, respectively. All these metabolites shared a E-resveratrol skeleton (5[(1E)-2-(4-Hydroxyphenyl) ethenyl]-1,3-benzenediol), either linked or not to a glycosidic moiety, suggesting a highly likely presence of these molecules in the extract. For cluster $\mathrm{C} 2$, a list of sugars was proposed by the database (Table 2), with the highest score obtained for saccharose (63\%). A list of terpenoids and tocopherols was proposed for cluster C3, but the obtained score values were relatively poor, all below $50 \%$ at this stage of the procedure. For cluster C4, 
1 a list of flavonoids was obtained, the top ranked molecules were (+)-catechin and taxifolin

2 derivatives with (+)-catechin having the highest score (52\%). Cluster C5 was a small cluster of

3 only $11 \mathrm{HMBC}$ correlations for which a list of various sugars with low score values was

4 obtained. This cluster contained in fact the $\mathrm{HMBC}$ correlation values of either a simple sugar

5 or the sugar moiety of a glycosylated molecule. Cluster C6 contained only 8 correlations that

6 did not allow prioritizing any relevant result.

7

Figure 4| HMBC-network obtained after NMR analysis of the bark extract of $P$. abies. Colors represent the different clusters: red $\mathrm{C} 1$, green $\mathrm{C2}$, blue $\mathrm{C} 3$, yellow $\mathrm{C} 4$, pink $\mathrm{C} 5$ and light blue C6.

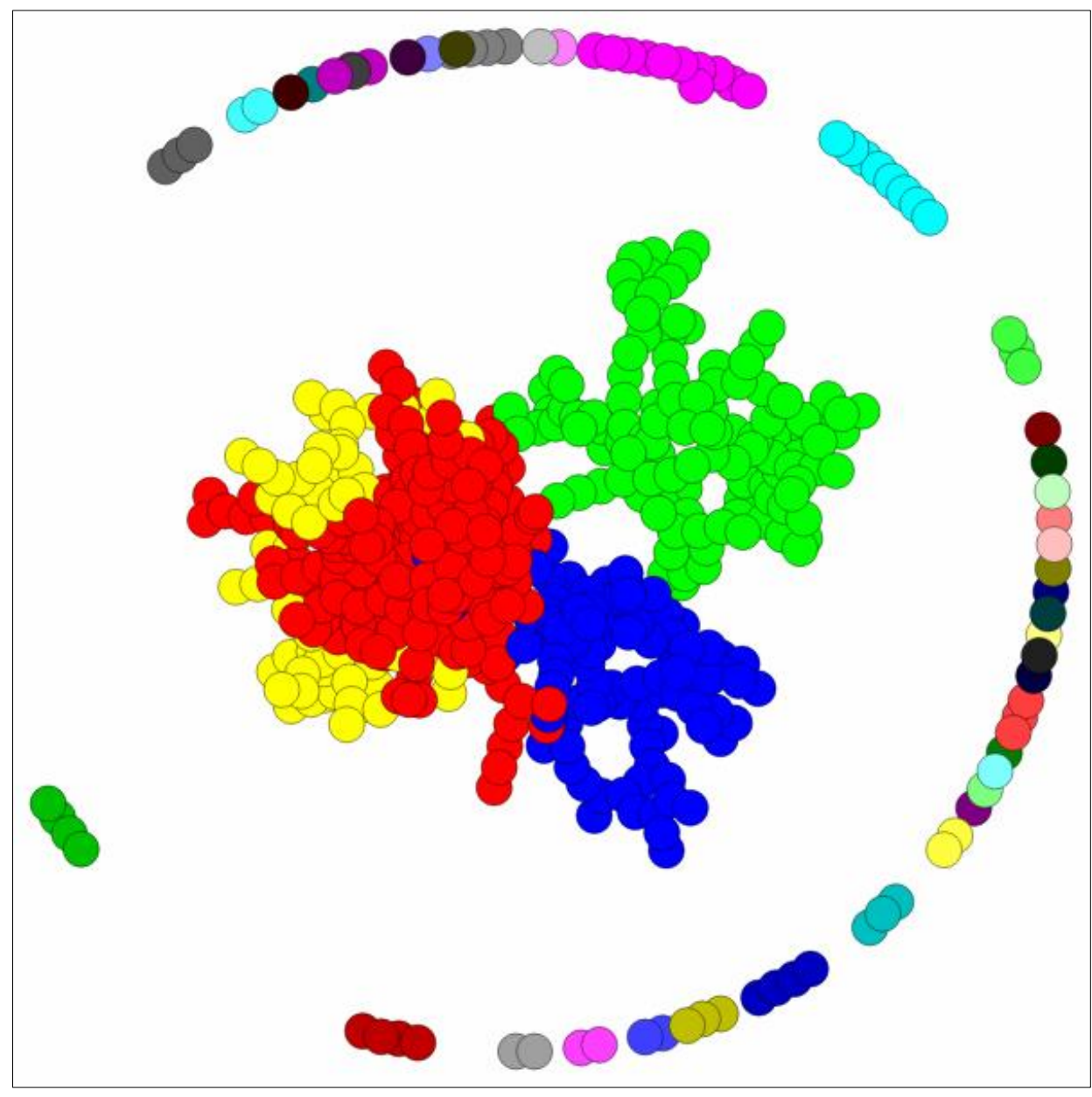


1 The search for clusters of $\mathrm{HMBC}$ correlations resulted in the identification of specific groups

2 of molecules. However, as the HMBC fingerprint of the molecules contained in the extract 3 were in some cases divided between several clusters, a second database search was performed for the 20 top ranked molecules of each cluster to compare their theoretical HMBC correlations to the total experimental $\mathrm{HMBC}$ correlations. As a result, a new list of molecules was obtained with improved scores, mainly containing stilbenes, flavonoids and sugars. The new scores of the top ranked molecules of cluster C1 increased, putting the glycosylated molecules in the top of the list with score values of $95 \%$ for E-isorhapontin, $94 \%$ for $E$-piceid, $91 \%$ for $E$-astringin, and $92 \%$ for $E$-rhaponticin. The scores of stilbene aglycones increased up to $81 \%$ for E-resveratrol, $82 \%$ for $E$-astringinin and $79 \% E$-rhapontigenin. The score of saccharose identified from cluster C2 also increased up to $74 \%$, and the score of 3,4dimethoxyphenyl- $\beta-D$-glucopyranoside, that was initially ranked at $12^{\text {th }}$ position in the cluster $\mathrm{C} 2$, arrived after the second step with a score value of $84 \%$. For cluster $\mathrm{C} 3$, the scores of the proposed molecules did not increased significantly after the second step of the database search, all remaining below $60 \%$. The score value of (+)-catechin identified in cluster C4 was increased up to $81 \%$, and other flavonoids including taxifolin, dihydrokaempferol, and taxifolin 3'-O- $\beta$-D-glucopyranoside arrived also in the top ranked molecules with score values of $87 \%, 86 \%$ and $85 \%$, respectively.

A final search was then performed to check the matching between the theoretical HSQC correlations of the top ranked molecules to the HSQC chemical shift values collected in the experimental spectrum. The final list of proposed molecules is given in Table $\mathbf{2}$, with fourteen molecules achieving a score value of $100 \%$. 
1 In summary, among the top ranked molecules obtained over the three step of our

2 identification procedure, fourteen molecules including $E$-isorhapontin, $E$-astringin, $E$-piceid,

3 saccharose, (+)-catechin, taxifolin, taxifolin 3'-O- $\beta$-D-glucopyranoside, 3,4-dimethoxyphenyl$\beta$-D-glucopyranoside, E-resveratrol, E-piceatannol, E-rhapontigenin, E-rhaponticin, maltose, and dihydrokaempferol were putatively identified in the methanol bark extract of $P$. abies (Table 2). In order to unambiguously validate the presence of these molecules in the extract, their 1D and 2D NMR data were manually checked by going back to the experimental 1D and 2D NMR spectra. The presence of eight compounds was fully confirmed (Fig. 6), namely Eisorhapontin, E-astringin, E-piceid, saccharose, (+)-catechin, taxifolin, taxifolin $3^{\prime}-O-\beta-D-$ glucopyranoside, and 3,4-dimethoxyphenyl- $\beta$-D-glucopyranoside. E-resveratrol was easily eliminated from the list of hits because no $\mathrm{HMBC}$ correlation corresponding to the highly characteristic symmetrical part of the 1,3-benzenediol moiety of the resveratrol carbon skeleton was detected, a correlation that is always present in all the spectra of compounds bearing this structural feature and that is due to the existence of a strong $3 \mathrm{~J}$ coupling (J.Am. Chem. Soc., 1969, 91 (17), pp 4940-4941, pas mieux...). Similarly, E-piceatannol and Erhapontigenin were also eliminated from the list of hits because no HMBC correlation corresponding to the symmetrical part of the 1,3-benzenediol moiety was detected by NMR. Concerning $E$-rhaponticin, which is a positional isomer of $E$-isorhapontin, the manual checking of experimental NMR data for the validation of E-isorhapontin enabled to discard $E$ rhaponticin from the list of hits thanks to three main indices (Fig. 5): First, we confirmed via the interpretation of the $\mathrm{HMBC}$ spectrum of the crude extract that the methoxy group of the putatively identified E-rhaponticin was directly linked to C-1 at $147.7 \mathrm{ppm}$. Secondly, the proton at $6.99 \mathrm{ppm}$ linked to $\mathrm{C}-4$ at $119.9 \mathrm{ppm}$ exhibited $\mathrm{HMBC}$ correlations with $\mathrm{C}-2$ at 146.4 ppm, with C-3 at 114.8 ppm, with C-6 at 109.1 ppm, but not with C-1 at $147.7 \mathrm{ppm}$. An 
$1 \mathrm{HMBC}$ correlation was also observed between the proton at 7.14 linked to C-6 and C-1 at 147.7

2 ppm, indicating that $\mathrm{C} 1$ belongs to the same carbon skeleton as C-2, C-3, C-4, and C-6.

3 Therefore C-1 and C-4 were placed in a para position from each other. 2 Thirdly, the 4 experimental chemical shift value of C-3 was $114.8 \mathrm{ppm}$, a value which is very close to the 5 predicted value of the same carbon position for $E$-isorhapontin at $115.0 \mathrm{ppm}$, while the same 6 carbon position of the $E$-rhaponticin is predicted at $110.6 \mathrm{ppm}$. Therefore the validation of the 7 structure of $E$-isorhapontin lead to discard the possible presence of its very similar isomer $E$ 8 rhaponticin in the methanol bark extract of $P$. abies. For dihydrokaempferol and maltose, the 9 experimental chemical shift values and 2D NMR connectivity data were not all confirmed after manual checking, therefore both compounds were also discarded from the list of hits. 
1 Figure 5 | Experimental HMBC correlations observed for E-isorhapontin. Chemical shift

2 values: $\mathrm{H} 4 \rightarrow \mathrm{C} 2$ (6.99 ppm $\rightarrow 146.4 \mathrm{ppm}) ; \mathrm{H} 4 \rightarrow \mathrm{C} 3$ (6.99 ppm $\rightarrow 114.8 \mathrm{ppm}), \mathrm{H} 4 \rightarrow \mathrm{C} 6$ (6.99 ppm,

$\left.3 \quad{ }^{13} \mathrm{C} 109.1 \mathrm{ppm}\right), \mathrm{H} 6 \rightarrow \mathrm{C} 4(7.14 \mathrm{ppm} \rightarrow 119.9 \mathrm{ppm}), \mathrm{H} 6 \rightarrow \mathrm{C} 1(7.14 \mathrm{ppm} \rightarrow 147.7 \mathrm{ppm}), \mathrm{H} 6 \rightarrow \mathrm{C} 2$

$4 \quad$ (7.14 ppm $\rightarrow 146.4 \mathrm{ppm}), \mathrm{H} 8 \rightarrow \mathrm{C} 1(3.9 \mathrm{ppm} \rightarrow 147.7 \mathrm{ppm})$.

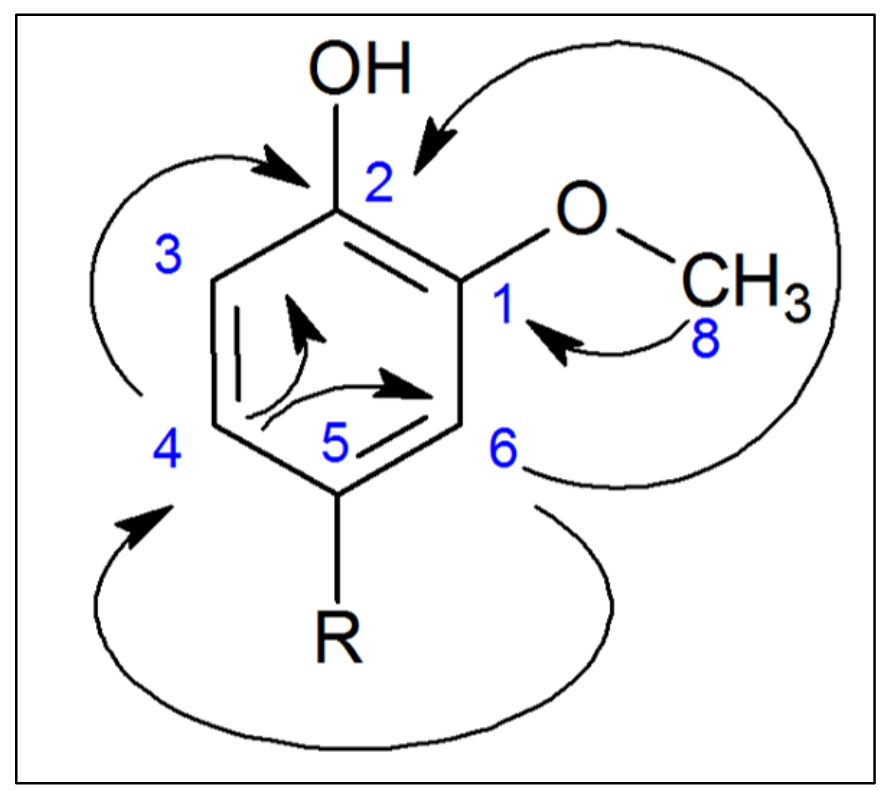

8 Table 2. Proposal list for the P. abies bark extract.

\begin{tabular}{|c|c|c|c|c|c|}
\hline & Putatively identified metabolites & $\begin{array}{l}\text { Score } 1 \text { (HMBC } \\
\text { cluster only) }\end{array}$ & $\begin{array}{l}\text { Score } 2 \text { (HMBC } \\
\text { full spectrum) }\end{array}$ & $\begin{array}{l}\text { Score } \\
\text { HSQC }\end{array}$ & Validation \\
\hline C1 & $\begin{array}{l}\text { E-resveratrol } \\
\text { E-piceid } \\
\text { E-isorhapontin } \\
\text { E-piceatannol (astringinin) } \\
\text { E-rhapontigenin } \\
\text { E-pterostilbene } \\
\text { E-astringin } \\
7,3 \text { '-dihydroxy-4'-methoxy- } \\
\text { isoflavone } \\
\text { rhaponticin } \\
\text { E-resveratrol-3-O-D-glucuronide }\end{array}$ & $\begin{array}{l}76 \\
76 \\
73 \\
71 \\
69 \\
68 \\
66 \\
66 \\
\\
65 \\
64 \\
\end{array}$ & $\begin{array}{l}81 \\
94 \\
95 \\
82 \\
79 \\
73 \\
91 \\
66 \\
\\
92 \\
79\end{array}$ & $\begin{array}{l}100 \\
100 \\
100 \\
100 \\
100 \\
91 \\
100 \\
75 \\
100 \\
93 \\
\end{array}$ & $\begin{array}{l}\text { eliminated* } \\
\text { confirmed } \\
\text { confirmed } \\
\text { eliminated* } \\
\text { eliminated* } \\
\text { eliminated } \\
\text { confirmed } \\
\text { eliminated } \\
\text { eliminated* } \\
\text { eliminated }\end{array}$ \\
\hline
\end{tabular}




\begin{tabular}{|c|c|c|c|c|c|}
\hline $\mathrm{C} 2$ & $\begin{array}{l}\text { saccharose } \\
\text { maltose } \\
\text { sophorose } \\
\text { myricetin 3-O-rutinoside } \\
\text { kojibiose } \\
\text { kaempferol 3,4'-diglucoside } \\
\text { rutinose } \\
\text { vanillic acid glucoside } \\
\text { laricitrin 3-O-glucoside } \\
\text { Laricitrin 3-O-rutinoside }\end{array}$ & $\begin{array}{l}63 \\
59 \\
59 \\
57 \\
57 \\
54 \\
53 \\
53 \\
52 \\
52\end{array}$ & $\begin{array}{l}74 \\
72 \\
65 \\
70 \\
65 \\
61 \\
62 \\
63 \\
62 \\
65\end{array}$ & $\begin{array}{l}100 \\
100 \\
79 \\
82 \\
86 \\
75 \\
92 \\
91 \\
67 \\
67\end{array}$ & $\begin{array}{l}\text { confirmed } \\
\text { eliminated* } \\
\text { eliminated } \\
\text { eliminated } \\
\text { eliminated } \\
\text { eliminated } \\
\text { eliminated } \\
\text { eliminated } \\
\text { eliminated } \\
\text { eliminated }\end{array}$ \\
\hline C3 & $\begin{array}{l}\text { 13-epimanool } \\
\text { cycloisosativene } \\
\text { neointermedeol } \\
\text { veticadinol } \\
\text { phylloquinone } \\
\text { docosadienoic acid } \\
\text { gamma-tocopherol } \\
\text { beta-tocopherol } \\
\text { delta-tocopherol } \\
\text { alpha-tocopherol }\end{array}$ & $\begin{array}{l}47 \\
41 \\
39 \\
39 \\
39 \\
39 \\
38 \\
37 \\
36 \\
35\end{array}$ & $\begin{array}{l}58 \\
49 \\
59 \\
55 \\
55 \\
54 \\
58 \\
60 \\
57 \\
52\end{array}$ & $\begin{array}{l}44 \\
69 \\
58 \\
50 \\
44 \\
89 \\
48 \\
45 \\
55 \\
42\end{array}$ & $\begin{array}{l}\text { eliminated } \\
\text { eliminated } \\
\text { eliminated } \\
\text { eliminated } \\
\text { eliminated } \\
\text { eliminated } \\
\text { eliminated } \\
\text { eliminated } \\
\text { eliminated } \\
\text { eliminated }\end{array}$ \\
\hline C4 & $\begin{array}{l}\text { (+)-catechin } \\
\text { taxifolin } \\
\text { (-)-epicatechin } \\
\text { syringetin } \\
\text { (+)-catechin gallate } \\
\text { dihydrokaempferol } \\
\text { (-)-epigallocatechin } \\
\text { taxifolin 3'-O-glucoside } \\
\text { epi-afzelechin } \\
\text { flavogallonic acid }\end{array}$ & $\begin{array}{l}52 \\
41 \\
40 \\
40 \\
37 \\
36 \\
33 \\
31 \\
30 \\
30\end{array}$ & $\begin{array}{l}81 \\
88 \\
62 \\
55 \\
81 \\
86 \\
51 \\
85 \\
48 \\
40\end{array}$ & $\begin{array}{l}100.0 \\
100.0 \\
88.9 \\
50.0 \\
72.7 \\
100.0 \\
75.0 \\
100.0 \\
70.0 \\
50.0\end{array}$ & $\begin{array}{l}\text { confirmed } \\
\text { confirmed } \\
\text { eliminated } \\
\text { eliminated } \\
\text { eliminated } \\
\text { eliminated* } \\
\text { eliminated } \\
\text { confirmed } \\
\text { eliminated } \\
\text { eliminated }\end{array}$ \\
\hline C5 & $\begin{array}{l}\text { monogalloylglucose } \\
\text { lactose } \\
\text { maltose } \\
\text { laricitrin 3-O-glucoside } \\
\text { 3,3'-di-O-methylellagic acid 4'-O- } \\
\text { beta-D-xylopyranoside } \\
\text { myricetin 3-O-glucoside } \\
\text { 3,3',4-tri- } O \text {-methylellagic acid-4'-O- } \\
\text { beta-D-glucopyranoside } \\
\text { quercetin-3-O-alpha-D- } \\
\text { arabinopyranoside } \\
\text { cibarian } \\
\text { 3,4-dimethoxyphenyl- } \beta \text {-D- } \\
\text { glucopyranoside }\end{array}$ & $\begin{array}{l}21 \\
20 \\
20 \\
19 \\
19 \\
\\
18 \\
17 \\
\\
17\end{array}$ & $\begin{array}{l}76 \\
60 \\
72 \\
62 \\
53 \\
\\
70 \\
47 \\
48 \\
\end{array}$ & $\begin{array}{l}78 \\
86 \\
100 \\
67 \\
80 \\
\\
82 \\
67 \\
55 \\
27 \\
100\end{array}$ & $\begin{array}{l}\text { eliminated } \\
\text { eliminated } \\
\text { eliminated* } \\
\text { eliminated } \\
\text { eliminated } \\
\text { eliminated } \\
\text { eliminated } \\
\text { eliminated } \\
\text { eliminated } \\
\text { confirmed }\end{array}$ \\
\hline
\end{tabular}


Figure 6 | Chemical structures of the eight confirmed metabolites in the $P$. abies bark extract. Colors highlighted atoms with chemical shifts that matched chemical shift values from the experimental $\mathrm{HMBC}$ peak list; Pink: matching molecular features, Blue: matching both ${ }^{1} \mathrm{H}$ and ${ }^{13} \mathrm{C}$ chemical shifts, Green: matching only ${ }^{13} \mathrm{C}$ chemical shifts, Red: Matching only ${ }^{1} \mathrm{H}$ chemical shifts.

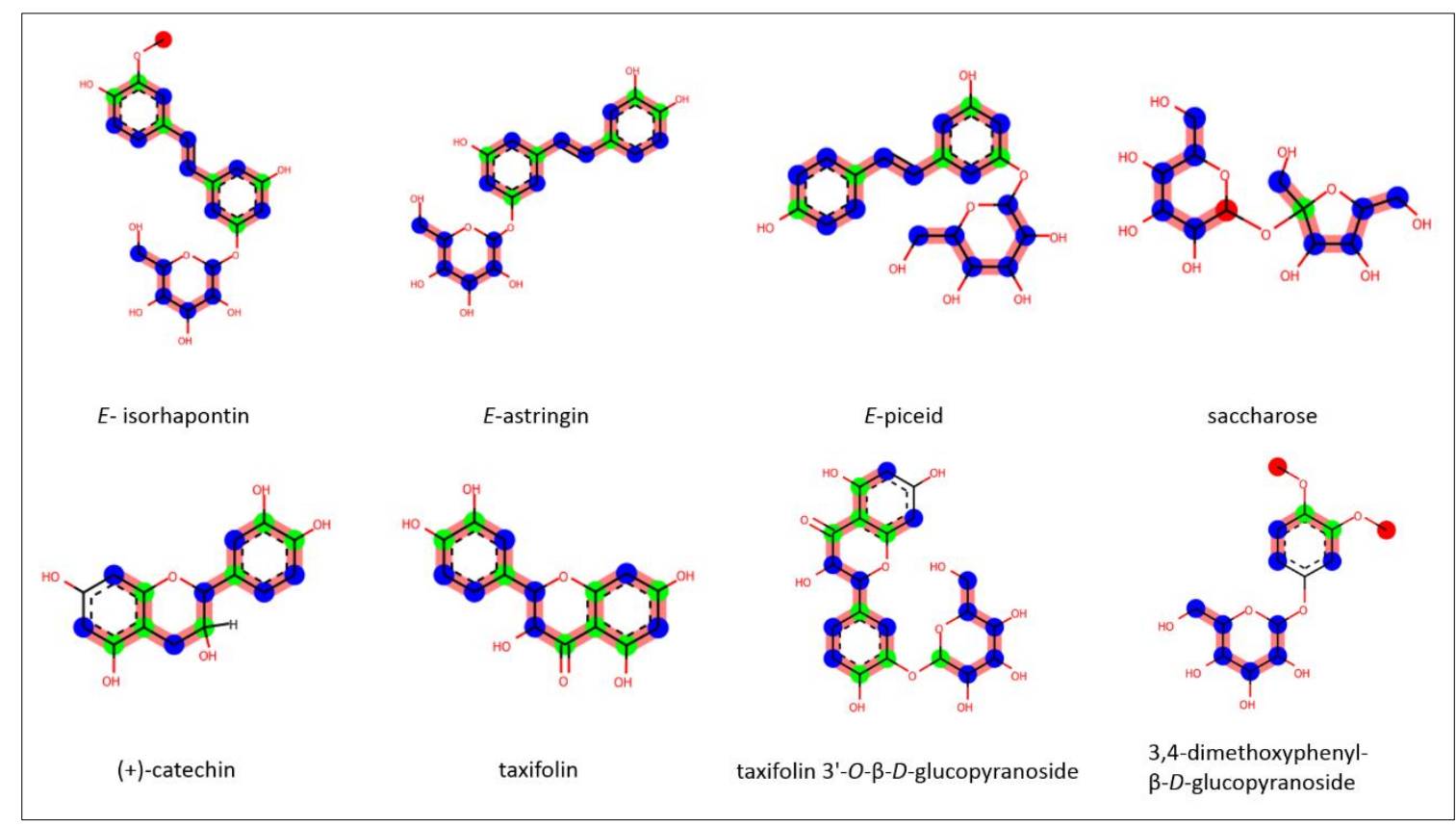

\section{Conclusion}

A new method exploiting $\mathrm{HMBC}$ and $\mathrm{HSQC}$ correlation data was developed in order to assist and accelerate the identification of natural metabolites directly within mixtures, while increasing the identification accuracy thanks to the richness of the information brought by $\mathrm{HMBC}$ and $\mathrm{HSQC}$ spectra. To the best of our knowledge, this is the first application of the network theory for the exploitation of heteronuclear 2D NMR data in the context of natural mixture analysis. In this paper, the proof of concept of the method was illustrated by two samples, a simplified model mixture of three commercial compounds and a real life natural extract. In the crude methanol extract of the bark of Picea abies, the method allowed the accurate identification of 8 molecules, namely $E$ - 
1 isorhapontin, E-astringin, E-piceid, saccharose, (+)-catechin, taxifolin, taxifolin $3^{\prime}-O-\beta-D-$

2 glucopyranoside and 3,4-dimethoxyphenyl-O- $\beta$-D-glucopyranoside. This method achieves

3 a better performance when applied to the analysis of simplified fractions with well

4 resolved $\mathrm{HMBC}$ spectra. The performance of this method is indeed highly dependent on

5 the HMBC spectrum quality because the latter determines the reliability of the initial peak

6 picking step. The method allows an early stage chemical profiling of the natural extracts

7 and it could be very complementary to MS-based dereplication by combining the different

8 advantages of each method in order to cross validate the results and have more accurate

9 insight into the chemical composition of the extracts. 